\title{
Worst-Case Optimal Redistribution of VCG Payments
}

\author{
Mingyu Guo \\ Duke University \\ Department of Computer Science \\ Durham, NC, USA \\ mingyu@cs.duke.edu
}

\author{
Vincent Conitzer \\ Duke University \\ Department of Computer Science \\ Durham, NC, USA \\ conitzer@cs.duke.edu
}

\begin{abstract}
For allocation problems with one or more items, the wellknown Vickrey-Clarke-Groves (VCG) mechanism is efficient, strategy-proof, individually rational, and does not incur a deficit. However, the VCG mechanism is not (strongly) budget balanced: generally, the agents' payments will sum to more than 0 . If there is an auctioneer who is selling the items, this may be desirable, because the surplus payment corresponds to revenue for the auctioneer. However, if the items do not have an owner and the agents are merely interested in allocating the items efficiently among themselves, any surplus payment is undesirable, because it will have to flow out of the system of agents. In 2006, Cavallo [3] proposed a mechanism that redistributes some of the VCG payment back to the agents, while maintaining efficiency, strategy-proofness, individual rationality, and the non-deficit property. In this paper, we extend this result in a restricted setting. We study allocation settings where there are multiple indistinguishable units of a single good, and agents have unit demand. (For this specific setting, Cavallo's mechanism coincides with a mechanism proposed by Bailey in 1997 [2].) Here we propose a family of mechanisms that redistribute some of the VCG payment back to the agents. All mechanisms in the family are efficient, strategyproof, individually rational, and never incur a deficit. The family includes the Bailey-Cavallo mechanism as a special case. We then provide an optimization model for finding the optimal mechanism - that is, the mechanism that maximizes redistribution in the worst case -inside the family, and show how to cast this model as a linear program. We give both numerical and analytical solutions of this linear program, and the (unique) resulting mechanism shows significant improvement over the Bailey-Cavallo mechanism (in the worst case). Finally, we prove that the obtained mechanism is optimal among all anonymous deterministic mechanisms that satisfy the above properties.
\end{abstract}

Permission to make digital or hard copies of all or part of this work for personal or classroom use is granted without fee provided that copies are not made or distributed for profit or commercial advantage and that copies bear this notice and the full citation on the first page. To copy otherwise, to republish, to post on servers or to redistribute to lists, requires prior specific permission and/or a fee.

EC'07, June 13-16, 2007, San Diego, California, USA.

Copyright 2007 ACM 978-1-59593-653-0/07/0006 ...\$5.00.

\section{Categories and Subject Descriptors}

J.4 [Computer Applications]: Social and Behavioral Sciences-Economics; I.2.11 [Distributed Artificial Intelligence]: Multiagent Systems

\section{General Terms}

Algorithms, Economics, Theory

\section{Keywords}

Mechanism Design, Vickrey-Clarke-Groves Mechanism, Payment Redistribution

\section{INTRODUCTION}

Many important problems in computer science and electronic commerce can be modeled as resource allocation problems. In such problems, we want to allocate the resources (or items) to the agents that value them the most. Unfortunately, agents' valuations are private knowledge, and self-interested agents will lie about their valuations if this is to their benefit. One solution is to auction off the items, possibly in a combinatorial auction where agents can bid on bundles of items. There exist ways of determining the payments that the agents make in such an auction that incentivizes the agents to report their true valuations - that is, the payments make the auction strategy-proof. One very general way of doing so is to use the VCG mechanism [23, 4,12 . (The VCG mechanism is also known as the Clarke mechanism or, in the specific context of auctions, the Generalized Vickrey Auction.)

Besides strategy-proofness, the VCG mechanism has several other nice properties in the context of resource allocation problems. It is efficient: the chosen allocation always maximizes the sum of the agents' valuations. It is also (expost) individually rational: participating in the mechanism never makes an agent worse off than not participating. Finally, it has a no-deficit property: the sum of the agents' payments is always nonnegative.

In many settings, another property that would be desirable is (strong) budget balance, meaning that the payments sum to exactly 0. Suppose the agents are trying to distribute some resources among themselves that do not have a previous owner. For example, the agents may be trying to allocate the right to use a shared good on a given day. Or, the agents may be trying to allocate a resource that they have collectively constructed, discovered, or otherwise obtained. If the agents use an auction to allocate these resources, and the sum of the agents' payments in the auction is positive, then this surplus payment must leave the system 
of the agents (for example, the agents must give the money to an outside party, or burn it). Naïve redistribution of the surplus payment (e.g. each of the $n$ agents receives $1 / n$ of the surplus) will generally result in a mechanism that is not strategy-proof (e.g. in a Vickrey auction, the second-highest bidder would want to increase her bid to obtain a larger redistribution payment). Unfortunately, the VCG mechanism is not budget balanced: typically, there is surplus payment. Unfortunately, in general settings, it is in fact impossible to design mechanisms that satisfy budget balance in addition to the other desirable properties $[16,11,21]$.

In light of this impossibility result, several authors have obtained budget balance by sacrificing some of the other desirable properties $[2,6,22,5]$. Another approach that is perhaps preferable is to use a mechanism that is "more" budget balanced than the VCG mechanism, and maintains all the other desirable properties. One way of trying to design such a mechanism is to redistribute some of the VCG payment back to the agents in a way that will not affect the agents' incentives (so that strategy-proofness is maintained), and that will maintain the other properties. In 2006, Cavallo [3] pursued exactly this idea, and designed a mechanism that redistributes a large amount of the total VCG payment while maintaining all of the other desirable properties of the VCG mechanism. For example, in a single-item auction (where the VCG mechanism coincides with the second-price sealed-bid auction), the amount redistributed to bidder $i$ by Cavallo's mechanism is $1 / n$ times the second-highest bid among bids other than $i$ 's bid. The total redistributed is at most the second-highest bid overall, and the redistribution to agent $i$ does not affect $i$ 's incentives because it does not depend on $i$ 's own bid.

In this paper, we restrict our attention to a limited setting, and in this setting we extend Cavallo's result. We study allocation settings where there are multiple indistinguishable units of a single good, and all agents have unit demand, i.e. they want only a single unit. For this specific setting, Cavallo's mechanism coincides with a mechanism proposed by Bailey in 1997 [2]. Here we propose the family of linear VCG redistribution mechanisms. All mechanisms in this family are efficient, strategy-proof, individually rational, and never incur a deficit. The family includes the Bailey-Cavallo mechanism as a special case (with the caveat that we only study allocation settings with multiple indistinguishable units of a single good and unit demand, while Bailey's and Cavallo's mechanisms can be applied outside these settings as well). We then provide an optimization model for finding the optimal mechanism inside the family, based on worst-case analysis. Both numerical and analytical solutions of this model are provided, and the resulting mechanism shows significant improvement over the BaileyCavallo mechanism (in the worst case). For example, for the problem of allocating a single unit, when the number of agents is 10, our mechanism always redistributes more than $98 \%$ of the total VCG payment back to the agents (whereas the Bailey-Cavallo mechanism redistributes only $80 \%$ in the worst case). Finally, we prove that our mechanism is in fact optimal among all anonymous deterministic mechanisms (even nonlinear ones) that satisfy the desirable properties.

Around the same time, the same mechanism has been in- dependently derived by Moulin [19]. ${ }^{1}$ Moulin actually pursues a different objective (also based on worst-case analysis): whereas our objective is to maximize the percentage of VCG payments that are redistributed, Moulin tries to minimize the overall payments from agents as a percentage of efficiency. It turns out that the resulting mechanisms are the same. Towards the end of this paper, we consider dropping the individual rationality requirement, and show that this does not change the optimal mechanism for our objective. For Moulin's objective, dropping individual rationality does change the optimal mechanism (but only if there are multiple units).

\section{PROBLEM DESCRIPTION}

Let $n$ denote the number of agents, and let $m$ denote the number of units. We only consider the case where $m<n$ (otherwise the problem becomes trivial). We also assume that $m$ and $n$ are always known. (This assumption is not harmful: in environments where anyone can join the auction, running a redistribution mechanism is typically not a good idea anyway, because everyone would want to join to collect part of the redistribution.)

Let the set of agents be $\left\{a_{1}, a_{2}, \ldots, a_{n}\right\}$, where $a_{i}$ is the agent with $i$ th highest report value $\hat{v}_{i}$ - that is, we have $\hat{v}_{1} \geq$ $\hat{v}_{2} \geq \ldots \geq \hat{v}_{n} \geq 0$. Let $v_{i}$ denote the true value of $a_{i}$. Given that the mechanism is strategy-proof, we can assume $v_{i}=\hat{v}_{i}$.

Under the VCG mechanism, each agent among $a_{1}, \ldots, a_{m}$ wins a unit, and pays $\hat{v}_{m+1}$ for this unit. Thus, the total VCG payment equals $m \hat{v}_{m+1}$. When $m=1$, this is the second-price or Vickrey auction.

We modify the mechanism as follows. After running the original VCG mechanism, the center returns to each agent $a_{i}$ some amount $z_{i}$, agent $a_{i}$ 's redistribution payment. We do not allow $z_{i}$ to depend on $\hat{v}_{i}$; because of this, $a_{i}$ 's incentives are unaffected by this redistribution payment, and the mechanism remains strategy-proof.

\section{LINEAR VCG REDISTRIBUTION MECHANISMS}

We are now ready to introduce the family of linear VCG redistribution mechanisms. Such a mechanism is defined by a vector of constants $c_{0}, c_{1}, \ldots, c_{n-1}$. The amount that the mechanism returns to agent $a_{i}$ is $z_{i}=c_{0}+c_{1} \hat{v}_{1}+c_{2} \hat{v}_{2}+$ $\ldots+c_{i-1} \hat{v}_{i-1}+c_{i} \hat{v}_{i+1}+\ldots+c_{n-1} \hat{v}_{n}$. That is, an agent receives $c_{0}$, plus $c_{1}$ times the highest bid other than the agent's own bid, plus $c_{2}$ times the second-highest other bid, etc. The mechanism is strategy-proof, because for all $i, z_{i}$ is independent of $\hat{v}_{i}$. Also, the mechanism is anonymous.

It is helpful to see the entire list of redistribution payments:

$z_{1}=c_{0}+c_{1} \hat{v}_{2}+c_{2} \hat{v}_{3}+c_{3} \hat{v}_{4}+\ldots+c_{n-2} \hat{v}_{n-1}+c_{n-1} \hat{v}_{n}$
$z_{2}=c_{0}+c_{1} \hat{v}_{1}+c_{2} \hat{v}_{3}+c_{3} \hat{v}_{4}+\ldots+c_{n-2} \hat{v}_{n-1}+c_{n-1} \hat{v}_{n}$
$z_{3}=c_{0}+c_{1} \hat{v}_{1}+c_{2} \hat{v}_{2}+c_{3} \hat{v}_{4}+\ldots+c_{n-2} \hat{v}_{n-1}+c_{n-1} \hat{v}_{n}$
$z_{4}=c_{0}+c_{1} \hat{v}_{1}+c_{2} \hat{v}_{2}+c_{3} \hat{v}_{3}+\ldots+c_{n-2} \hat{v}_{n-1}+c_{n-1} \hat{v}_{n}$
$\ldots$
$z_{i}=c_{0}+c_{1} \hat{v}_{1}+c_{2} \hat{v}_{2}+\ldots+c_{i-1} \hat{v}_{i-1}+c_{i} \hat{v}_{i+1}+\ldots+c_{n-1} \hat{v}_{n}$
$\ldots$
$z_{n-2}=c_{0}+c_{1} \hat{v}_{1}+c_{2} \hat{v}_{2}+c_{3} \hat{v}_{3}+\ldots+c_{n-2} \hat{v}_{n-1}+c_{n-1} \hat{v}_{n}$
$z_{n-1}=c_{0}+c_{1} \hat{v}_{1}+c_{2} \hat{v}_{2}+c_{3} \hat{v}_{3}+\ldots+c_{n-2} \hat{v}_{n-2}+c_{n-1} \hat{v}_{n}$
$z_{n}=c_{0}+c_{1} \hat{v}_{1}+c_{2} \hat{v}_{2}+c_{3} \hat{v}_{3}+\ldots+c_{n-2} \hat{v}_{n-2}+c_{n-1} \hat{v}_{n-1}$

${ }^{1}$ We thank Rakesh Vohra for pointing us to Moulin's working paper. 
Not all choices of the constants $c_{0}, \ldots, c_{n-1}$ produce a mechanism that is individually rational, and not all choices of the constants produce a mechanism that never incurs a deficit. Hence, to obtain these properties, we need to place some constraints on the constants.

To satisfy the individual rationality criterion, each agent's utility should always be non-negative. An agent that does not win a unit obtains a utility that is equal to the agent's redistribution payment. An agent that wins a unit obtains a utility that is equal to the agent's valuation for the unit, minus the VCG payment $\hat{v}_{m+1}$, plus the agent's redistribution payment.

Consider agent $a_{n}$, the agent with the lowest bid. Since this agent does not win an item $(m<n)$, her utility is just her redistribution payment $z_{n}$. Hence, for the mechanism to be individually rational, the $c_{i}$ must be such that $z_{n}$ is always nonnegative. If the $c_{i}$ have this property, then it actually follows that $z_{i}$ is nonnegative for every $i$, for the following reason. Suppose there exists some $i<n$ and some vector of bids $\hat{v}_{1} \geq \hat{v}_{2} \geq \ldots \geq \hat{v}_{n} \geq 0$ such that $z_{i}<0$. Then, consider the bid vector that results from replacing $\hat{v}_{j}$ by $\hat{v}_{j+1}$ for all $j \geq i$, and letting $\hat{v}_{n}=0$. If we omit $\hat{v}_{n}$ from this vector, the same vector results that results from omitting $\hat{v}_{i}$ from the original vector. Therefore, $a_{n}$ 's redistribution payment under the new vector should be the same as $a_{i}$ 's redistribution payment under the old vector-but this payment is negative.

If all redistribution payments are always nonnegative, then the mechanism must be individually rational (because the VCG mechanism is individually rational, and the redistribution payment only increases an agent's utility). Therefore, the mechanism is individually rational if and only if for any bid vector, $z_{n} \geq 0$.

To satisfy the non-deficit criterion, the sum of the redistribution payments should be less than or equal to the total VCG payment. So for any bid vector $\hat{v}_{1} \geq \hat{v}_{2} \geq \ldots \geq \hat{v}_{n} \geq$ 0 , the constants $c_{i}$ should make $z_{1}+z_{2}+\ldots+z_{n} \leq m \hat{v}_{m+1}$.

We define the family of linear VCG redistribution mechanisms to be the set of all redistribution mechanisms corresponding to constants $c_{i}$ that satisfy the above constraints (so that the mechanisms will be individually rational and have the no-deficit property). We now give two examples of mechanisms in this family.

Example 1 (Bailey-Cavallo mechanism): Consider the mechanism corresponding to $c_{m+1}=\frac{m}{n}$ and $c_{i}=0$ for all other $i$. Under this mechanism, each agent receives a redistribution payment of $\frac{m}{n}$ times the $(m+1)$ th highest bid from another agent. Hence, $a_{1}, \ldots, a_{m+1}$ receive a redistribution payment of $\frac{m}{n} \hat{v}_{m+2}$, and the others receive $\frac{m}{n} \hat{v}_{m+1}$. Thus, the total redistribution payment is $(m+1) \frac{m}{n} \hat{v}_{m+2}+(n-m-$ 1) $\frac{m}{n} \hat{v}_{m+1}$. This redistribution mechanism is individually rational, because all the redistribution payments are nonnegative, and never incurs a deficit, because $(m+1) \frac{m}{n} \hat{v}_{m+2}+$ $(n-m-1) \frac{m}{n} \hat{v}_{m+1} \leq n \frac{m}{n} \hat{v}_{m+1}=m \hat{v}_{m+1}$. (We note that for this mechanism to make sense, we need $n \geq m+2$.)

Example 2: Consider the mechanism corresponding to $c_{m+1}=\frac{m}{n-m-1}, c_{m+2}=-\frac{m(m+1)}{(n-m-1)(n-m-2)}$, and $c_{i}=0$ for all other $i$. In this mechanism, each agent receives a redistribution payment of $\frac{m}{n-m-1}$ times the $(m+1)$ th highest reported value from other agents, minus $\frac{m(m+1)}{(n-m-1)(n-m-2)}$ times the $(m+2)$ th highest reported value from other agents. Thus, the total redistribution payment is $m \hat{v}_{m+1}-$ $\frac{m(m+1)(m+2)}{(n-m-1)(n-m-2)} \hat{v}_{m+3}$. If $n \geq 2 m+3$ (which is equivalent to $\left.\frac{m}{n-m-1} \geq \frac{m(m+1)}{(n-m-1)(n-m-2)}\right)$, then each agent always receives a nonnegative redistribution payment, thus the mechanism is individually rational. Also, the mechanism never incurs a deficit, because the total VCG payment is $m \hat{v}_{m+1}$, which is greater than the amount $m \hat{v}_{m+1}-\frac{m(m+1)(m+2)}{(n-m-1)(n-m-2)} \hat{v}_{m+3}$ that is redistributed.

Which of these two mechanisms is better? Is there another mechanism that is even better? This is what we study in the next section.

\section{OPTIMAL REDISTRIBUTION MECHANISMS}

Among all linear VCG redistribution mechanisms, we would like to be able to identify the one that redistributes the greatest percentage of the total VCG payment. ${ }^{2}$ This is not a well-defined notion: it may be that one mechanism redistributes more on some bid vectors, and another more on other bid vectors. We emphasize that we do not assume that a prior distribution over bidders' valuations is available, so we cannot compare them based on expected redistribution. Below, we study three well-defined ways of comparing redistribution mechanisms: best-case performance, dominance, and worst-case performance.

Best-case performance. One way of evaluating a mechanism is by considering the highest redistribution percentage that it achieves. Consider the previous two examples. For the first example, the total redistribution payment is $(m+1) \frac{m}{n} \hat{v}_{m+2}+(n-m-1) \frac{m}{n} \hat{v}_{m+1}$. When $\hat{v}_{m+2}=\hat{v}_{m+1}$, this is equal to the total VCG payment $m \hat{v}_{m+1}$. Thus, this mechanism redistributes $100 \%$ of the total VCG payment in the best case. For the second example, the total redistribution payment is $m \hat{v}_{m+1}-\frac{m(m+1)(m+2)}{(n-m-1)(n-m-2)} \hat{v}_{m+3}$. When $\hat{v}_{m+3}=0$, this is equal to the total VCG payment $m \hat{v}_{m+1}$. Thus, this mechanism also redistributes $100 \%$ of the total VCG payment in the best case.

Moreover, there are actually infinitely many mechanisms that redistribute $100 \%$ of the total VCG payment in the best case - for example, any convex combination of the above two will redistribute $100 \%$ if both $\hat{v}_{m+2}=\hat{v}_{m+1}$ and $\hat{v}_{m+3}=0$.

Dominance. Inside the family of linear VCG redistribution mechanisms, we say one mechanism dominates another mechanism if the first one redistributes at least as much as the other for any bid vector. For the previous two examples, neither dominates the other, because they each redistribute $100 \%$ in different cases. It turns out that there is no mechanism in the family that dominates all other mechanisms in the family. For suppose such a mechanism exists. Then, it should dominate both examples above. Consider the remaining VCG payment (the VCG payment failed to be redistributed). The remaining VCG payment of the dominant mechanism should be 0 whenever $\hat{v}_{m+2}=\hat{v}_{m+1}$ or $\hat{v}_{m+3}=0$. Now, the remaining VCG payment is a linear function of the $\hat{v}_{i}$ (linear redistribution), and therefore also a polynomial function. The above implies that this function can be written as $\left(\hat{v}_{m+2}-\hat{v}_{m+1}\right)\left(\hat{v}_{m+3}\right) P\left(\hat{v}_{1}, \hat{v}_{2}, \ldots, \hat{v}_{n}\right)$, where $P$ is a

\footnotetext{
${ }^{2}$ The percentage redistributed seems the natural criterion to use, among other things because it is scale-invariant: if we multiply all bids by the same positive constant (for example, if we change the units by re-expressing the bids in euros instead of dollars), we would not want the behavior of our mechanism to change.
} 
polynomial function. But since the function must be linear (has degree at most 1 ), it follows that $P=0$. Thus, a dominant mechanism would always redistribute all of the VCG payment, which is not possible. (If it were possible, then our worst-case optimal redistribution mechanism would also always redistribute all of the VCG payment, and we will see later that it does not.)

Worst-case performance. Finally, we can evaluate a mechanism by considering the lowest redistribution percentage that it guarantees. For the first example, the total redistribution payment is $(m+1) \frac{m}{n} \hat{v}_{m+2}+(n-m-1) \frac{m}{n} \hat{v}_{m+1}$, which is greater than or equal to $(n-m-1) \frac{m}{n} \hat{v}_{m+1}$. So in the worst case, which is when $\hat{v}_{m+2}=0$, the percentage redistributed is $\frac{n-m-1}{n}$. For the second example, the total redistribution payment is $m \hat{v}_{m+1}-\frac{m(m+1)(m+2)}{(n-m-1)(n-m-2)} \hat{v}_{m+3}$, which is greater than or equal to $m \hat{v}_{m+1}\left(1-\frac{(m+1)(m+2)}{(n-m-1)(n-m-2)}\right)$. So in the worst case, which is when $\hat{v}_{m+3}=\hat{v}_{m+1}$, the percentage redistributed is $1-\frac{(m+1)(m+2)}{(n-m-1)(n-m-2)}$. Since we assume that the number of agents $n$ and the number of units $m$ are known, we can determine which example mechanism has better worst-case performance by comparing the two quantities. When $n=6$ and $m=1$, for the first example (Bailey-Cavallo mechanism), the percentage redistributed in the worst case is $\frac{2}{3}$, and for the second example, this percentage is $\frac{1}{2}$, which implies that for this pair of $n$ and $m$, the first mechanism has better worst-case performance. On the other hand, when $n=12$ and $m=1$, for the first example, the percentage redistributed in the worst case is $\frac{5}{6}$, and for the second example, this percentage is $\frac{14}{15}$, which implies that this time the second mechanism has better worst-case performance.

Thus, it seems most natural to compare mechanisms by the percentage of total VCG payment that they redistribute in the worst case. This percentage is undefined when the total VCG payment is 0 . To deal with this, technically, we define the worst-case redistribution percentage as the largest $k$ so that the total amount redistributed is at least $k$ times the total VCG payment, for all bid vectors. (Hence, as long as the total amount redistributed is at least 0 when the total VCG payment is 0 , these cases do not affect the worst-case percentage.) This corresponds to the following optimization problem:

Maximize $k$ (the percentage redistributed in the
worst case)
Subject to:
For every bid vector $\hat{v}_{1} \geq \hat{v}_{2} \geq \ldots \geq \hat{v}_{n} \geq 0$
$z_{n} \geq 0$ (individual rationality)
$z_{1}+z_{2}+\ldots+z_{n} \leq m \hat{v}_{m+1}$ (non-deficit)
$z_{1}+z_{2}+\ldots+z_{n} \geq k m \hat{v}_{m+1}$ (worst-case constraint)
We recall that $z_{i}=c_{0}+c_{1} \hat{v}_{1}+c_{2} \hat{v}_{2}+\ldots+c_{i-1} \hat{v}_{i-1}+$
$c_{i} \hat{v}_{i+1}+\ldots+c_{n-1} \hat{v}_{n}$.

\section{TRANSFORMATION TO LINEAR PROGRAMMING}

The optimization problem given in the previous section can be rewritten as a linear program, based on the following observations.

Claim 1. If $c_{0}, c_{1}, \ldots, c_{n-1}$ satisfy both the individual rationality and the non-deficit constraints, then $c_{i}=0$ for $i=0, \ldots, m$.
Proof. First, let us prove that $c_{0}=0$. Consider the bid vector in which $\hat{v}_{i}=0$ for all $i$. To obtain individual rationality, we must have $c_{0} \geq 0$. To satisfy the non-deficit constraint, we must have $c_{0} \leq 0$. Thus we know $c_{0}=0$. Now, if $c_{i}=0$ for all $i$, there is nothing to prove. Otherwise, let $j=\min \left\{i \mid c_{i} \neq 0\right\}$. Assume that $j \leq m$. We recall that we can write the individual rationality constraint as follows: $z_{n}=c_{0}+c_{1} \hat{v}_{1}+c_{2} \hat{v}_{2}+c_{3} \hat{v}_{3}+\ldots+c_{n-2} \hat{v}_{n-2}+c_{n-1} \hat{v}_{n-1} \geq 0$ for any bid vector. Let us consider the bid vector in which $\hat{v}_{i}=1$ for $i \leq j$ and $\hat{v}_{i}=0$ for the rest. In this case $z_{n}=c_{j}$, so we must have $c_{j} \geq 0$. The non-deficit constraint can be written as follows: $z_{1}+z_{2}+\ldots+z_{n} \leq m \hat{v}_{m+1}$ for any bid vector. Consider the same bid vector as above. We have $z_{i}=0$ for $i \leq j$, because for these bids, the $j$ th highest other bid has value 0 , so all the $c_{i}$ that are nonzero are multiplied by 0 . For $i>j$, we have $z_{i}=c_{j}$, because the $j$ th highest other bid has value 1 , and all lower bids have value 0 . So the non-deficit constraint tells us that $c_{j}(n-j) \leq m \hat{v}_{m+1}$. Because $j \leq m, \hat{v}_{m+1}=0$, so the right hand side is 0 . We also have $n-j>0$ because $j \leq m<n$. So $c_{j} \leq 0$. Because we have already established that $c_{j} \geq 0$, it follows that $c_{j}=0$; but this is contrary to assumption. So $j>m$.

Incidentally, this claim also shows that if $m=n-1$, then $c_{i}=0$ for all $i$. Thus, we are stuck with the VCG mechanism. From here on, we only consider the case where $m<n-1$.

Claim 2. The individual rationality constraint can be written as follows: $\sum_{i=m+1}^{j} c_{i} \geq 0$ for $j=m+1, \ldots, n-1$.

Before proving this claim, we introduce the following lemma.

Lemma 1. Given a positive integer $k$ and a set of real constants $s_{1}, s_{2}, \ldots, s_{k},\left(s_{1} t_{1}+s_{2} t_{2}+\ldots+s_{k} t_{k} \geq 0\right.$ for any $\left.t_{1} \geq t_{2} \geq \ldots \geq t_{k} \geq 0\right)$ if and only if $\left(\sum_{i=1}^{j} s_{i} \geq 0\right.$ for $j=1,2, \ldots, k)$.

Proof. Let $d_{i}=t_{i}-t_{i+1}$ for $i=1,2, \ldots, k-1$, and $d_{k}=$ $t_{k}$. Then $\left(s_{1} t_{1}+s_{2} t_{2}+\ldots+s_{k} t_{k} \geq 0\right.$ for any $t_{1} \geq t_{2} \geq \ldots \geq$ $\left.t_{k} \geq 0\right)$ is equivalent to $\left(\left(\sum_{i=1}^{1} s_{i}\right) d_{1}+\left(\sum_{i=1}^{2} s_{i}\right) d_{2}+\ldots+\right.$ $\left(\sum_{i=1}^{k} s_{i}\right) d_{k} \geq 0$ for any set of arbitrary non-negative $\left.d_{j}\right)$. When $\sum_{i=1}^{j} s_{i} \geq 0$ for $j=1,2, \ldots, k$, the above inequality is obviously true. If for some $j, \sum_{i=1}^{j} s_{i}<0$, if we set $d_{j}>0$ and $d_{i}=0$ for all $i \neq j$, then the above inequality becomes false. So $\sum_{i=1}^{j} s_{i} \geq 0$ for $j=1,2, \ldots, k$ is both necessary and sufficient. $\square$

We are now ready to present the proof of Claim 2 .

Proof. The individual rationality constraint can be written as $z_{n}=c_{0}+c_{1} \hat{v}_{1}+c_{2} \hat{v}_{2}+c_{3} \hat{v}_{3}+\ldots+c_{n-2} \hat{v}_{n-2}+$ $c_{n-1} \hat{v}_{n-1} \geq 0$ for any bid vector $\hat{v}_{1} \geq \hat{v}_{2} \geq \ldots \geq \hat{v}_{n-1} \geq$ $\hat{v}_{n} \geq 0$. We have already shown that $c_{i}=0$ for $i \leq m$. Thus, the above can be simplified to $z_{n}=c_{m+1} \hat{v}_{m+1}+$ $c_{m+2} \hat{v}_{m+2}+\ldots+c_{n-2} \hat{v}_{n-2}+c_{n-1} \hat{v}_{n-1} \geq 0$ for any bid vector. By the above lemma, this is equivalent to $\sum_{i=m+1}^{j} c_{i} \geq 0$ for $j=m+1, \ldots, n-1$.

Claim 3. The non-deficit constraint and the worst-case constraint can also be written as linear inequalities involving only the $c_{i}$ and $k$.

Proof. The non-deficit constraint requires that for any bid vector, $z_{1}+z_{2}+\ldots+z_{n} \leq m \hat{v}_{m+1}$, where $z_{i}=c_{0}+c_{1} \hat{v}_{1}+$ 
$c_{2} \hat{v}_{2}+\ldots+c_{i-1} \hat{v}_{i-1}+c_{i} \hat{v}_{i+1}+\ldots+c_{n-1} \hat{v}_{n}$ for $i=1,2, \ldots, n$. Because $c_{i}=0$ for $i \leq m$, we can simplify this inequality to

$$
\begin{aligned}
& q_{m+1} \hat{v}_{m+1}+q_{m+2} \hat{v}_{m+2}+\ldots+q_{n} \hat{v}_{n} \geq 0 \\
& q_{m+1}=m-(n-m-1) c_{m+1} \\
& q_{i}=-(i-1) c_{i-1}-(n-i) c_{i}, \text { for } i=m+2, \ldots, n-1 \text { (when } \\
& m+2>n-1, \text { this set of equalities is empty) } \\
& q_{n}=-(n-1) c_{n-1}
\end{aligned}
$$

By the above lemma, this is equivalent to $\sum_{i=m+1}^{j} q_{i} \geq 0$ for $j=m+1, \ldots, n$. So, we can simplify further as follows:

$$
\begin{aligned}
& q_{m+1} \geq 0 \Longleftrightarrow(n-m-1) c_{m+1} \leq m \\
& q_{m+1}+\ldots+q_{m+i} \geq 0 \Longleftrightarrow n \sum_{j=m+1}^{j=m+i-1} c_{j}+(n-m- \\
& i) c_{m+i} \leq m \text { for } i=2, \ldots, n-m-1 \\
& q_{m+1}+\ldots+q_{n} \geq 0 \Longleftrightarrow n \sum_{j=m+1}^{j=n-1} c_{j} \leq m
\end{aligned}
$$

So, the non-deficit constraint can be written as a set of linear inequalities involving only the $c_{i}$.

The worst-case constraint can be also written as a set of linear inequalities, by the following reasoning. The worstcase constraint requires that for any bid input $z_{1}+z_{2}+\ldots+$ $z_{n} \geq k m \hat{v}_{m+1}$, where $z_{i}=c_{0}+c_{1} \hat{v}_{1}+c_{2} \hat{v}_{2}+\ldots+c_{i-1} \hat{v}_{i-1}+$ $c_{i} \hat{v}_{i+1}+\ldots+c_{n-1} \hat{v}_{n}$ for $i=1,2, \ldots, n$. Because $c_{i}=0$ for $i \leq m$, we can simplify this inequality to

$$
\begin{aligned}
& Q_{m+1} \hat{v}_{m+1}+Q_{m+2} \hat{v}_{m+2}+\ldots+Q_{n} \hat{v}_{n} \geq 0 \\
& Q_{m+1}=(n-m-1) c_{m+1}-k m \\
& Q_{i}=(i-1) c_{i-1}+(n-i) c_{i}, \text { for } i=m+2, \ldots, n-1 \\
& Q_{n}=(n-1) c_{n-1}
\end{aligned}
$$

By the above lemma, this is equivalent to $\sum_{i=m+1}^{j} Q_{i} \geq 0$ for $j=m+1, \ldots, n$. So, we can simplify further as follows:

$$
\begin{aligned}
& Q_{m+1} \geq 0 \Longleftrightarrow(n-m-1) c_{m+1} \geq k m \\
& Q_{m+1}+\ldots+Q_{m+i} \geq 0 \Longleftrightarrow n \sum_{j=m+1}^{j=m+i-1} c_{j}+(n-m- \\
& i) c_{m+i} \geq k m \text { for } i=2, \ldots, n-m-1 \\
& Q_{m+1}+\ldots+Q_{n} \geq 0 \Longleftrightarrow n \sum_{j=m+1}^{j=n-1} c_{j} \geq k m
\end{aligned}
$$

So, the worst-case constraint can also be written as a set of linear inequalities involving only the $c_{i}$ and $k$.

Combining all the claims, we see that the original optimization problem can be transformed into the following linear program.

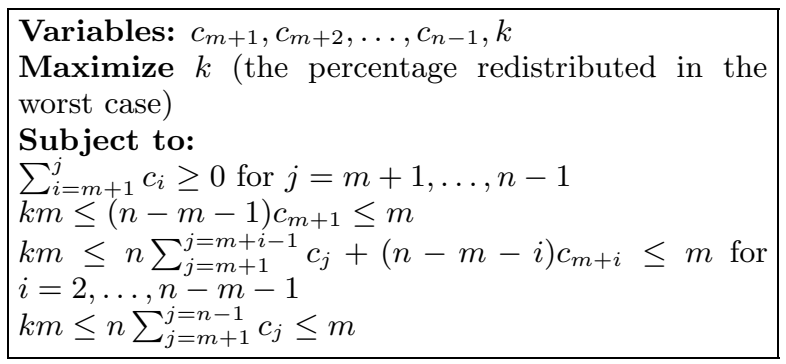

\section{NUMERICAL RESULTS}

For selected values of $n$ and $m$, we solved the linear program using Glpk (GNU Linear Programming Kit). In the table below, we present the results for a single unit $(m=1)$. We present $1-k$ (the percentage of the total VCG payment that is not redistributed by the worst-case optimal mechanism in the worst case) instead of $k$ in the second column because writing $k$ would require too many significant digits. Correspondingly, the third column displays the percentage

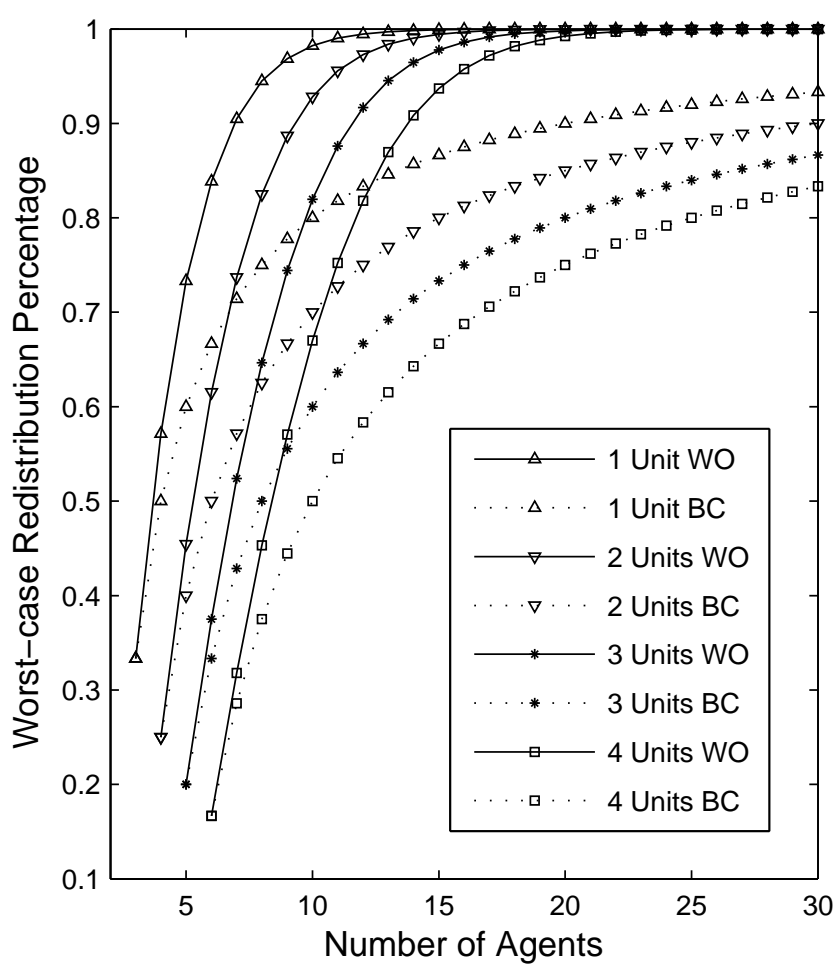

Figure 1: A comparison of the worst-case optimal mechanism (WO) and the Bailey-Cavallo mechanism (BC).

of the total VCG payment that is not redistributed by the Bailey-Cavallo mechanism in the worst case (which is equal to $\left.\frac{2}{n}\right)$.

$\begin{array}{ccc}n & 1-k & \text { Bailey - Cavallo Mechanism } \\ 3 & 66.7 \% & 66.7 \% \\ 4 & 42.9 \% & 50.0 \% \\ 5 & 26.7 \% & 40.0 \% \\ 6 & 16.1 \% & 33.3 \% \\ 7 & 9.52 \% & 28.6 \% \\ 8 & 5.51 \% & 25.0 \% \\ 9 & 3.14 \% & 22.2 \% \\ 10 & 1.76 \% & 20.0 \% \\ 20 & 3.62 e-5 & 10.0 \% \\ 30 & 5.40 e-8 & 6.67 e-2 \\ 40 & 7.09 e-11 & 5.00 e-2\end{array}$

The worst-case optimal mechanism significantly outperforms the Bailey-Cavallo mechanism in the worst case. Perhaps more surprisingly, the worst-case optimal mechanism sometimes does better in the worst case than the BaileyCavallo mechanism does on average, as the following example shows.

Recall that the total redistribution payment of the BaileyCavallo mechanism is $(m+1) \frac{m}{n} \hat{v}_{m+2}+(n-m-1) \frac{m}{n} \hat{v}_{m+1}$. For the single-unit case, this simplifies to $\frac{2}{n} \hat{v}_{3}+\frac{n-2}{n} \hat{v}_{2}$. Hence the percentage of the total VCG payment that is not redistributed is $\frac{\hat{v}_{2}-\frac{2}{n} \hat{v}_{3}-\frac{n-2}{n} \hat{v}_{2}}{\hat{v}_{2}}=\frac{2}{n}-\frac{2}{n} \frac{\hat{v}_{3}}{\hat{v}_{2}}$, which has an expected value of $E\left(\frac{2}{n}-\frac{2}{n} \frac{\hat{v}_{3}}{\hat{v}_{2}}\right)=\frac{2}{n}-\frac{2}{n} E \frac{\hat{v}_{3}}{\hat{v}_{2}}$. Suppose the bid values are drawn from a uniform distribution over $[0,1]$. The theory of order statistics tells us that the 
joint probability density function of $\hat{v}_{2}$ and $\hat{v}_{3}$ is $f\left(\hat{v}_{3}, \hat{v}_{2}\right)=$ $n(n-1)(n-2) \hat{v}_{3}^{n-3}\left(1-\hat{v}_{2}\right)$ for $\hat{v}_{2} \geq \hat{v}_{3}$. Now, $E \frac{\hat{v}_{3}}{\hat{v}_{2}}=$ $\int_{0}^{1} \int_{0}^{\hat{v}_{2}} \frac{\hat{v}_{3}}{\hat{v}_{2}} f\left(\hat{v}_{3}, \hat{v}_{2}\right) d \hat{v}_{3} d \hat{v}_{2}=\frac{n-2}{n-1}$. So, the expected value of the remaining percentage is $\frac{2}{n}-\frac{2}{n} \frac{n-2}{n-1}=\frac{2}{n(n-1)}$. For $n=20$, this is $5.26 e-3$, whereas the remaining percentage for the worst-case optimal mechanism is $3.62 e-5$ in the worst case.

Let us present the optimal solution for the case $n=5$ in detail. By solving the above linear program, we find that the optimal values for the $c_{i}$ are $c_{2}=\frac{11}{45}, c_{3}=-\frac{1}{9}$, and $c_{4}=\frac{1}{15}$. That is, the redistribution payment received by each agent is: $\frac{11}{45}$ times the second highest bid among the other agents, minus $\frac{1}{9}$ times the third highest bid among the other agents, plus $\frac{1}{15}$ times the fourth highest bid among the other agents.

The total amount redistributed is $\frac{11}{15} \hat{v}_{2}+\frac{4}{15} \hat{v}_{3}-\frac{4}{15} \hat{v}_{4}+$ $\frac{4}{15} \hat{v}_{5}$; in the worst case, $\frac{11}{15} \hat{v}_{2}$ is redistributed. Hence, the percentage of the total VCG payment that is not redistributed is never more than $\frac{4}{15}=26.7 \%$.

Finally, we compare the worst-case optimal mechanism to the Bailey-Cavallo mechanism for $m=1,2,3,4, n=m+$ $2, \ldots, 30$. These results are in Figure 1.

We see that for any $m$, when $n=m+2$, the worst-case optimal mechanism has the same worst-case performance as the Bailey-Cavallo mechanism (actually, in this case, the worst-case optimal mechanism is identical to the BaileyCavallo mechanism). When $n>m+2$, the worst-case optimal mechanism outperforms the Bailey-Cavallo mechanism (in the worst case).

\section{ANALYTICAL CHARACTERIZATION OF THE WORST-CASE OPTIMAL MECHANISM}

We recall that our linear program has the following form:

Variables: $c_{m+1}, c_{m+2}, \ldots, c_{n-1}, k$
Maximize $k$ (the percentage redistributed in the
worst case)
Subject to:
$\sum_{i=m+1}^{j} c_{i} \geq 0$ for $j=m+1, \ldots, n-1$
$k m \leq(n-m-1) c_{m+1} \leq m$
$k m \leq n \sum_{j=m+i-1}^{j=m+1} c_{j}+(n-m-i) c_{m+i} \leq m$ for
$i=2, \ldots, n-m-1$
$k m \leq n \sum_{j=m+1}^{j=n-1} c_{j} \leq m$

A linear program has no solution if and only if either the objective is unbounded, or the constraints are contradictory (there is no feasible solution). It is easy to see that $k$ is bounded above by 1 (redistributing more than $100 \%$ violates the non-deficit constraint). Also, a feasible solution always exists, for example, $k=0$ and $c_{i}=0$ for all $i$. So an optimal solution always exists. Observe that the linear program model depends only on the number of agents $n$ and the number of units $m$. Hence the optimal solution is a function of $n$ and $m$. It turns out that this optimal solution can be analytically characterized as follows.

THEOREM 1. For any $m$ and $n$ with $n \geq m+2$, the worstcase optimal mechanism (among linear $\bar{V} C G$ redistribution mechanisms) is unique. For this mechanism, the percentage redistributed in the worst case is

$$
k^{*}=1-\frac{\left(\begin{array}{c}
n-1 \\
m
\end{array}\right)}{\sum_{j=m}^{n-1}\left(\begin{array}{c}
n-1 \\
j
\end{array}\right)}
$$

The worst-case optimal mechanism is characterized by the following values for the $c_{i}$ :

$$
c_{i}^{*}=\frac{(-1)^{i+m-1}(n-m)\left(\begin{array}{c}
n-1 \\
m-1
\end{array}\right)}{i \sum_{j=m}^{n-1}\left(\begin{array}{c}
n-1 \\
j
\end{array}\right)} \frac{1}{\left(\begin{array}{c}
n-1 \\
i
\end{array}\right)} \sum_{j=i}^{n-1}\left(\begin{array}{c}
n-1 \\
j
\end{array}\right)
$$

for $i=m+1, \ldots, n-1$.

It should be noted that we have proved $c_{i}=0$ for $i \leq m$ in Claim 1.

PROOF. We first rewrite the linear program as follows. We introduce new variables $x_{m+1}, x_{m+2}, \ldots, x_{n-1}$, defined by $x_{j}=\sum_{i=m+1}^{j} c_{i}$ for $j=m+1, \ldots, n-1$. The linear program then becomes:

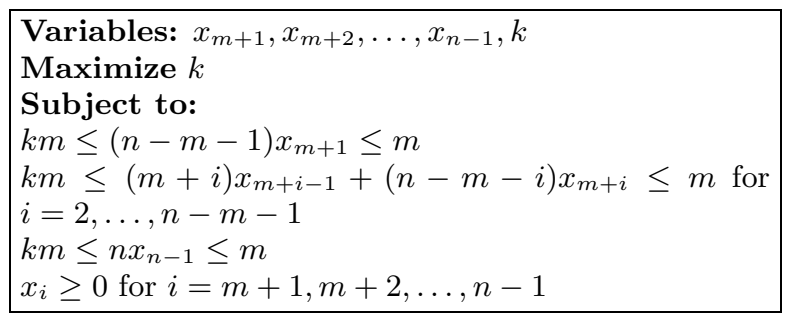

We will prove that for any optimal solution to this linear program, $k=k^{*}$. Moreover, we will prove that when $k=k^{*}$, $x_{j}=\sum_{i=m+1}^{j} c_{i}^{*}$ for $j=m+1, \ldots, n-1$. This will prove the theorem.

We first make the following observations:

$(n-m-1) c_{m+1}^{*}$

$$
\begin{aligned}
& =(n-m-1) \frac{(n-m)\left(\begin{array}{c}
n-1 \\
m-1
\end{array}\right)}{(m+1) \sum_{j=m}^{n-1}\left(\begin{array}{c}
n-1 \\
j
\end{array}\right)} \frac{1}{\left(\begin{array}{c}
n-1 \\
m+1
\end{array}\right)} \sum_{j=m+1}^{n-1}\left(\begin{array}{c}
n-1 \\
j
\end{array}\right) \\
& =(n-m-1) \frac{(n-m)\left(\begin{array}{c}
n-1 \\
m-1
\end{array}\right)}{(m+1) \sum_{j=m}^{n-1}\left(\begin{array}{c}
n-1 \\
j
\end{array}\right)} \frac{1}{\left(\begin{array}{c}
n-1 \\
m+1
\end{array}\right)}\left(\sum_{j=m}^{n-1}\left(\begin{array}{c}
n-1 \\
j
\end{array}\right)-\left(\begin{array}{c}
n-1 \\
m
\end{array}\right)\right) \\
& =(n-m-1) \frac{m}{n-m-1}-(n-m-1) \frac{m\left(\begin{array}{c}
n-1 \\
m
\end{array}\right)}{(n-m-1) \sum_{j=m}^{n-1}\left(\begin{array}{c}
n-1 \\
j
\end{array}\right)} \\
& =m-\left(1-k^{*}\right) m=k^{*} m \\
& \text { For } i=m+1, \ldots, n-2 \text {, } \\
& i c_{i}^{*}+(n-i-1) c_{i+1}^{*}
\end{aligned}
$$

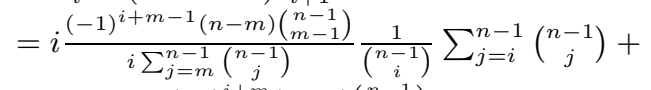

$$
\begin{aligned}
& (n-i-1) \frac{(-1)^{i+m}(n-m)\left(\begin{array}{c}
n-1 \\
m-1
\end{array}\right)}{(i+1) \sum_{j=m}^{n-1}\left(\begin{array}{c}
n-1 \\
j
\end{array}\right)} \frac{1}{\left(\begin{array}{c}
n-1 \\
i+1
\end{array}\right)} \sum_{j=i+1}^{n-1}\left(\begin{array}{c}
n-1 \\
j
\end{array}\right) \\
& =\frac{(-1)^{i+m-1}(n-m)\left(\begin{array}{c}
n-1 \\
m-1
\end{array}\right)}{\sum_{j=m}^{n-1}\left(\begin{array}{c}
n-1 \\
j
\end{array}\right)} \frac{1}{\left(\begin{array}{c}
n-1 \\
i
\end{array}\right)} \sum_{j=i}^{n-1}\left(\begin{array}{c}
n-1 \\
j
\end{array}\right)- \\
& (n-i-1) \frac{(-1)^{i+m-1}(n-m)\left(\begin{array}{c}
n-1 \\
m-1
\end{array}\right)}{(i+1) \sum_{j=m}^{n-1}\left(\begin{array}{c}
n-1 \\
j
\end{array}\right)} \frac{i+1}{\left(\begin{array}{c}
n-1 \\
i
\end{array}\right)(n-i-1)} \sum_{j=i+1}^{n-1}\left(\begin{array}{c}
n-1 \\
j
\end{array}\right) \\
& =\frac{(-1)^{i+m-1}(n-m)\left(\begin{array}{c}
n-1 \\
m-1
\end{array}\right)}{\sum_{j=m}^{n-1}\left(\begin{array}{c}
n-1 \\
j
\end{array}\right)} \\
& =(-1)^{i+m-1} m\left(1-k^{*}\right)
\end{aligned}
$$

Finally,

$(n-1) c_{n-1}^{*}$$$
=(n-1) \frac{(-1)^{n+m}(n-m)\left(\begin{array}{c}
n-1 \\
m-1
\end{array}\right)}{(n-1) \sum_{j=m}^{n-1}\left(\begin{array}{c}
n-1 \\
j
\end{array}\right)} \frac{1}{\left(\begin{array}{c}
n-1 \\
n-1
\end{array}\right)} \sum_{j=n-1}^{n-1}\left(\begin{array}{c}
n-1 \\
j
\end{array}\right)
$$$$
=(-1)^{m+n} m\left(1-k^{*}\right)
$$

Summarizing the above, we have:

$$
(n-m-1) c_{m+1}^{*}=k^{*} m
$$$$
(m+1) c_{m+1}^{*}+(n-m-2) c_{m+2}^{*}=m\left(1-k^{*}\right)
$$$$
(m+2) c_{m+2}^{*}+(n-m-3) c_{m+3}^{*}=-m\left(1-k^{*}\right)
$$$$
(m+3) c_{m+3}^{*}+(n-m-4) c_{m+4}^{*}=m\left(1-k^{*}\right)
$$ 
$(n-3) c_{n-3}^{*}+2 c_{n-2}^{*}=(-1)^{m+n-2} m\left(1-k^{*}\right)$

$(n-2) c_{n-2}^{*}+c_{n-1}^{*}=(-1)^{m+n-1} m\left(1-k^{*}\right)$

$(n-1) c_{n-1}^{*}=(-1)^{m+n} m\left(1-k^{*}\right)$

Let $x_{j}^{*}=\sum_{i=m+1}^{j} c_{i}^{*}$ for $j=m+1, m+2, \ldots, n-1$, the first equation in the above tells us that

$(n-m-1) x_{m+1}^{*}=k^{*} m$.

By adding the first two equations, we get

$(m+2) x_{m+1}^{*}+(n-m-2) x_{m+2}^{*}=m$

By adding the first three equations, we get

$(m+3) x_{m+2}^{*}+(n-m-3) x_{m+3}^{*}=k^{*} m$

By adding the first $i$ equations, where $i=2, \ldots, n-m-1$, we get

$(m+i) x_{m+i-1}^{*}+(n-m-i) x_{m+i}^{*}=m$ if $i$ is even

$(m+i) x_{m+i-1}^{*}+(n-m-i) x_{m+i}^{*}=k^{*} m$ if $i$ is odd

Finally by adding all the equations, we get

$n x_{n-1}^{*}=m$ if $n-m$ is even;

$n x_{n-1}^{*}=k^{*} m$ if $n-m$ is odd.

Thus, for all of the constraints other than the nonnegativity constraints, we have shown that they are satisfied by setting $x_{j}=x_{j}^{*}=\sum_{i=m+1}^{j} c_{i}^{*}$ and $k=k^{*}$. We next show that the nonnegativity constraints are satisfied by these settings as well.

For $m+1 \leq i, i+1 \leq n-1$, we have $\frac{1}{i} \frac{\sum_{j=i}^{n-1}\left(\begin{array}{c}n-1 \\ j\end{array}\right)}{\left(\begin{array}{c}n-1 \\ i\end{array}\right)}=\frac{1}{i} \sum_{j=i}^{n-1} \frac{i !(n-1-i) !}{j !(n-1-j) !} \geq \frac{1}{i+1} \sum_{j=i}^{n-2} \frac{i !(n-1-i) !}{j !(n-1-j) !} \geq$ $\frac{1}{i+1} \sum_{j=i}^{n-2} \frac{(i+1) !(n-1-i-1) !}{(j+1) !(n-1-j-1) !}=\frac{1}{i+1} \frac{\sum_{j=i+1}^{n-1}\left(\begin{array}{c}n-1 \\ j\end{array}\right)}{\left(\begin{array}{c}n-1 \\ i+1\end{array}\right)}$

This implies that the absolute value of $c_{i}^{*}$ is decreasing as $i$ increases (if the $c^{*}$ contains more than one number). We further observe that the $\operatorname{sign}$ of $c_{i}^{*}$ alternates, with the first element $c_{m+1}^{*}$ positive. So $x_{j}^{*}=\sum_{i=m+1}^{j} c_{i}^{*} \geq 0$ for all $j$. Thus, we have shown that these $x_{i}=x_{i}^{*}$ together with $k=k^{*}$ form a feasible solution of the linear program. We proceed to show that it is in fact the unique optimal solution.

First we prove the following claim:

Claim 4. If $\hat{k}, \hat{x}_{i}, i=m+1, m+2, \ldots, n-1$ satisfy the following inequalities:

$$
\begin{gathered}
\hat{k} m \leq(n-m-1) \hat{x}_{m+1} \leq m \\
\hat{k} m \leq(m+i) \hat{x}_{m+i-1}+(n-m-i) \hat{x}_{m+i} \leq m \text { for } \\
i=2, \ldots, n-m-1 \\
\hat{k} m \leq n \hat{x}_{n-1} \leq m \\
\hat{k} \geq k^{*}
\end{gathered}
$$

Then we must have that $\hat{x}_{i}=\hat{x}_{i}^{*}$ and $\hat{k}=k^{*}$.

Proof of Claim. Consider the first inequality. We know that $(n-m-1) x_{m+1}^{*}=k^{*} m$, so $(n-m-1) \hat{x}_{m+1} \geq \hat{k} m \geq$ $k^{*} m=(n-m-1) x_{m+1}^{*}$. It follows that $\hat{x}_{m+1} \geq x_{m+1}^{*}$ $(n-m-1 \neq 0)$.

Now, consider the next inequality for $i=2$. We know that $(m+2) x_{m+1}^{*}+(n-m-2) x_{m+2}^{*}=m$. It follows that $(n-m-2) \hat{x}_{m+2} \leq m-(m+2) \hat{x}_{m+1} \leq m-(m+2) x_{m+1}^{*}=$ $(n-m-2) x_{m+2}^{*}$, so $\hat{x}_{m+2} \leq x_{m+2}^{*}(i=2 \leq n-m-1 \Rightarrow$ $n-m-2 \neq 0)$.

Now consider the next inequality for $i=3$. We know that $(m+3) x_{m+2}^{*}+(n-m-3) x_{m+3}^{*}=m$. It follows that $(n-m-3) \hat{x}_{m+3} \geq \hat{k} m-(m+3) \hat{x}_{m+2} \geq k^{*} m-(m+3) x_{m+2}^{*}=$ $(n-m-3) x_{m+3}^{*}$, so $\hat{x}_{m+3} \geq x_{m+3}^{*}(i=3 \leq n-m-1 \Rightarrow$ $n-m-3 \neq 0)$.
Proceeding like this all the way up to $i=n-m-1$, we get that $\hat{x}_{m+i} \geq x_{m+i}^{*}$ if $i$ is odd and $\hat{x}_{m+i} \leq x_{m+i}^{*}$ if $i$ is even. Moreover, if one inequality is strict, then all subsequent inequalities are strict. Now, if we can prove $\hat{x}_{n-1}=x_{n-1}^{*}$, it would follow that the $x_{i}^{*}$ are equal to the $\hat{x}_{i}$ (which also implies that $\hat{k}=k^{*}$ ). We consider two cases:

Case 1: $n-m$ is even. We have: $n-m$ even $\Rightarrow n-m-1$ odd $\Rightarrow \hat{x}_{n-1} \geq x_{n-1}^{*}$. We also have: $n-m$ even $\Rightarrow n x_{n-1}^{*}=$ $m$. Combining these two, we get $m=n x_{n-1}^{*} \leq n \hat{x}_{n-1} \leq$ $m \Rightarrow \hat{x}_{n-1}=x_{n-1}^{*}$.

Case 2: $n-m$ is odd. In this case, we have $\hat{x}_{n-1} \leq x_{n-1}^{*}$, and $n x_{n-1}^{*}=k^{*} m$. Then, we have: $k^{*} m \leq \hat{k} m \leq n \hat{x}_{n-1} \leq$ $n x_{n-1}^{*}=k^{*} m \Rightarrow \hat{x}_{n-1}=x_{n-1}^{*}$.

This completes the proof of the claim.

It follows that if $\hat{k}, \hat{x}_{i}, i=m+1, m+2, \ldots, n-1$ is a feasible solution and $\hat{k} \geq k^{*}$, then since all the inequalities in Claim 4 are satisfied, we must have $\hat{x}_{i}=x_{i}^{*}$ and $\hat{k}=$ $k^{*}$. Hence no other feasible solution is as good as the one described in the theorem.

Knowing the analytical characterization of the worst-case optimal mechanism provides us with at least two major benefits. First, using these formulas is computationally more efficient than solving the linear program using a generalpurpose solver. Second, we can derive the following corollary.

COROllary 1. If the number of units $m$ is fixed, then as the number of agents $n$ increases, the worst-case percentage redistributed linearly converges to 1 , with a rate of convergence $\frac{1}{2}$. (That is, $\lim _{n \rightarrow \infty} \frac{1-k_{n+1}^{*}}{1-k_{n}^{*}}=\frac{1}{2}$. That is, in the limit, the percentage that is not redistributed halves for every additional agent.)

We note that this is consistent with the experimental data for the single-unit case, where the worst-case remaining percentage roughly halves each time we add another agent. The worst-case percentage that is redistributed under the Bailey-Cavallo mechanism also converges to 1 as the number of agents goes to infinity, but the convergence is much slower - it does not converge linearly (that is, letting $k_{n}^{C}$ be the percentage redistributed by the Bailey-Cavallo mechanism in the worst case for $n$ agents, $\lim _{n \rightarrow \infty} \frac{1-k_{n+1}^{C}}{1-k_{n}^{C}}=$ $\lim _{n \rightarrow \infty} \frac{n}{n+1}=1$ ). We now present the proof of the corollary.

Proof. When the number of agents is $n$, the worst-case percentage redistributed is $k_{n}^{*}=1-\frac{\left(\begin{array}{c}n-1 \\ m\end{array}\right)}{\sum_{j=m}^{n-1}\left(\begin{array}{c}n-1 \\ j\end{array}\right)}$. When the number of agents is $n+1$, the percentage becomes $k_{n+1}^{*}=$ $1-\frac{\left(\begin{array}{c}n \\ m\end{array}\right)}{\sum_{j=m}^{n}\left(\begin{array}{c}n \\ j\end{array}\right)}$. For $n$ sufficiently large, we will have $2^{n}-$ $m n^{m-1}>0$, and hence $\frac{1-k_{n+1}^{*}}{1-k_{n}^{*}}=\frac{\left(\begin{array}{c}n \\ m\end{array}\right) \sum_{j=m}^{n-1}\left(\begin{array}{c}n-1 \\ j\end{array}\right)}{\left(\begin{array}{c}n-1 \\ m\end{array}\right) \sum_{j=m}^{n}\left(\begin{array}{c}n \\ j\end{array}\right)}=$ $\frac{n}{n-m} \frac{2^{n-1}-\sum_{j=0}^{m-1}\left(\begin{array}{c}n-1 \\ j\end{array}\right)}{2^{n}-\sum_{j=0}^{m-1}\left(\begin{array}{c}n \\ j\end{array}\right)}$, and $\frac{n}{n-m} \frac{2^{n-1}-m(n-1)^{m-1}}{2^{n}} \leq \frac{1-k_{n+1}^{*}}{1-k_{n}^{*}}$ $\leq \frac{n}{n-m} \frac{2^{n-1}}{2^{n}-m n^{m-1}}$ (because $\left(\begin{array}{l}n \\ j\end{array}\right) \leq n^{i}$ if $\left.j \leq i\right)$.

$$
\text { Since we have }
$$$$
\lim _{n \rightarrow \infty} \frac{n}{n-m} \frac{2^{n-1}-m(n-1)^{m-1}}{2^{n}}=\frac{1}{2} \text {, and }
$$$$
\lim _{n \rightarrow \infty} \frac{n}{n-m} \frac{2^{n-1}}{2^{n}-m n^{m-1}}=\frac{1}{2} \text {, }
$$

it follows that $\lim _{n \rightarrow \infty} \frac{1-k_{n+1}^{*}}{1-k_{n}^{*}}=\frac{1}{2}$. 


\section{WORST-CASE OPTIMALITY OUTSIDE THE FAMILY}

In this section, we prove that the worst-case optimal redistribution mechanism among linear VCG redistribution mechanisms is in fact optimal (in the worst case) among all redistribution mechanisms that are deterministic, anonymous, strategy-proof, efficient and satisfy the non-deficit constraint. Thus, restricting our attention to linear VCG redistribution mechanisms did not come at a loss.

To prove this theorem, we need the following lemma. This lemma is not new: it was informally stated by Cavallo [3]. For completeness, we present it here with a detailed proof.

LEMma 2. A VCG redistribution mechanism is deterministic, anonymous and strategy-proof if and only if there exists a function $f: \mathbf{R}^{\mathbf{n}-\mathbf{1}} \rightarrow \mathbf{R}$, so that the redistribution payment $z_{i}$ received by $a_{i}$ satisfies

$$
z_{i}=f\left(\hat{v}_{1}, \hat{v}_{2}, \ldots, \hat{v}_{i-1}, \hat{v}_{i+1}, \ldots, \hat{v}_{n}\right)
$$

for all $i$ and all bid vectors.

ProOF. First, let us prove the "only if" direction, that is, if a VCG redistribution mechanism is deterministic, anonymous and strategy-proof then there exists a deterministic function $f: \mathbf{R}^{\mathbf{n}-1} \rightarrow \mathbf{R}$, which makes

$$
z_{i}=f\left(\hat{v}_{1}, \hat{v}_{2}, \ldots, \hat{v}_{i-1}, \hat{v}_{i+1}, \ldots, \hat{v}_{n}\right)
$$

for all $i$ and all bid vectors.

If a VCG redistribution mechanism is deterministic and anonymous, then for any bid vector $\hat{v}_{1} \geq \hat{v}_{2} \geq \ldots \geq \hat{v}_{n}$, the mechanism outputs a unique redistribution payment list: $z_{1}, z_{2}, \ldots, z_{n}$. Let $G: \mathbf{R}^{\mathbf{n}} \rightarrow \mathbf{R}^{\mathbf{n}}$ be the function that maps $\hat{v}_{1}, \hat{v}_{2}, \ldots, \hat{v}_{n}$ to $z_{1}, z_{2}, \ldots, z_{n}$ for all bid vectors. Let $H\left(i, x_{1}, x_{2}, \ldots, x_{n}\right)$ be the $i$ th element of $G\left(x_{1}, x_{2}, \ldots, x_{n}\right)$, so that $z_{i}=H\left(i, \hat{v}_{1}, \hat{v}_{2}, \ldots, \hat{v}_{n}\right)$ for all bid vectors and all $1 \leq i \leq n$. Because the mechanism is anonymous, two agents should receive the same redistribution payment if their bids are the same. So, if $\hat{v}_{i}=\hat{v}_{j}, H\left(i, \hat{v}_{1}, \hat{v}_{2}, \ldots, \hat{v}_{n}\right)=$ $H\left(j, \hat{v}_{1}, \hat{v}_{2}, \ldots, \hat{v}_{n}\right)$. Hence, if we let $j=\min \left\{t \mid \hat{v}_{t}=\hat{v}_{i}\right\}$, then $H\left(i, \hat{v}_{1}, \hat{v}_{2}, \ldots, \hat{v}_{n}\right)=H\left(j, \hat{v}_{1}, \hat{v}_{2}, \ldots, \hat{v}_{n}\right)$.

Let us define $K: \mathbf{R}^{\mathbf{n}} \rightarrow \mathbf{N} \times \mathbf{R}^{\mathbf{n}}$ as follows: $K\left(y, x_{1}, x_{2}\right.$, $\left.\ldots, x_{n-1}\right)=\left[j, w_{1}, w_{2}, \ldots, w_{n}\right]$, where $w_{1}, w_{2}, \ldots, w_{n}$ are $y, x_{1}, x_{2}, \ldots, x_{n-1}$ sorted in descending order, and $j=\min \left\{t \mid w_{t}=y\right\} . \quad\left(\left\{t \mid w_{t}=y\right\} \neq \emptyset\right.$ because $y \in\left\{w_{1}, w_{2}\right.$, $\left.\left.\ldots, w_{n}\right\}\right)$. Also let us define $F: \mathbf{R}^{\mathbf{n}} \rightarrow \mathbf{R}$ by

$F\left(\hat{v}_{i}, \hat{v}_{1}, \hat{v}_{2}, \ldots, \hat{v}_{i-1}, \hat{v}_{i+1}, \ldots, \hat{v}_{n}\right)$

$=H \circ K\left(\hat{v}_{i}, \hat{v}_{1}, \hat{v}_{2}, \ldots, \hat{v}_{i-1}, \hat{v}_{i+1}, \ldots, \hat{v}_{n}\right)$

$=H\left(\min \left\{t \mid \hat{v}_{t}=\hat{v}_{i}\right\}, \hat{v}_{1}, \hat{v}_{2}, \ldots, \hat{v}_{n}\right)$

$=H\left(i, \hat{v}_{1}, \hat{v}_{2}, \ldots, \hat{v}_{n}\right)=z_{i}$.

That is, $F$ is the redistribution payment to an agent that bids $\hat{v}_{i}$ when the other bids are $\hat{v}_{1}, \hat{v}_{2}, \ldots, \hat{v}_{i-1}, \hat{v}_{i+1}, \ldots, \hat{v}_{n}$.

Since our mechanism is required to be strategy-proof, and the space of valuations is unrestricted, $z_{i}$ should be independent of $\hat{v}_{i}$ by Lemma 1 in Cavallo [3]. Hence, we can simply ignore the first variable input to $F$; let $f\left(x_{1}, x_{2}, \ldots, x_{n-1}\right)=$ $F\left(0, x_{1}, x_{2}, \ldots, x_{n-1}\right)$. So, we have for all bid vectors and $i, z_{i}=f\left(\hat{v}_{1}, \hat{v}_{2}, \ldots, \hat{v}_{i-1}, \hat{v}_{i+1}, \ldots, \hat{v}_{n}\right)$. This completes the proof for the "only if" direction.

For the "if" direction, if the redistribution payment received by $a_{i}$ satisfies $z_{i}=f\left(\hat{v}_{1}, \hat{v}_{2}, \ldots, \hat{v}_{i-1}, \hat{v}_{i+1}, \ldots, \hat{v}_{n}\right)$ for all bid vectors and $i$, then this is clearly a deterministic and anonymous mechanism. To prove strategy-proofness, we observe that because an agent's redistribution payment is not affected by her own bid, her incentives are the same as in the VCG mechanism, which is strategy-proof.

Now we are ready to introduce the next theorem:

THEOREM 2. For any $m$ and $n$ with $n \geq m+2$, the worstcase optimal mechanism among the family of linear VCG redistribution mechanisms is worst-case optimal among all mechanisms that are deterministic, anonymous, strategy-proof, efficient and satisfy the non-deficit constraint.

While we needed individual rationality earlier in the paper, this theorem does not mention it, that is, we can not find a mechanism with better worst-case performance even if we sacrifice individual rationality. (The worst-case optimal linear VCG redistribution mechanism is of course individually rational.)

Proof. Suppose there is a redistribution mechanism (when the number of units is $m$ and the number of agents is $n$ ) that satisfies all of the above properties and has a better worstcase performance than the worst-case optimal linear VCG redistribution mechanism, that is, its worst-case redistribution percentage $\hat{k}$ is strictly greater than $k^{*}$.

By Lemma 2, for this mechanism, there is a function $f$ : $\mathbf{R}^{\mathbf{n}-\mathbf{1}} \rightarrow \mathbf{R}$ so that $z_{i}=f\left(\hat{v}_{1}, \hat{v}_{2}, \ldots, \hat{v}_{i-1}, \hat{v}_{i+1}, \ldots, \hat{v}_{n}\right)$ for all $i$ and all bid vectors. We first prove that $f$ has the following properties.

Claim 5. $f(1,1, \ldots, 1,0,0, \ldots, 0)=0$ if the number of $1 \mathrm{~s}$ is less than or equal to $\mathrm{m}$.

ProOF OF ClAIM. We assumed that for this mechanism, the worst-case redistribution percentage satisfies $\hat{k}>k^{*} \geq$ 0 . If the total VCG payment is $x$, the total redistribution payment should be in $[\hat{k} x, x]$ (non-deficit criterion). Consider the case where all agents bid 0 , so that the total VCG payment is also 0 . Hence, the total redistribution payment should be in $[\hat{k} \cdot 0,0]$ - that is, it should be 0 . Hence every agent's redistribution payment $f(0,0, \ldots, 0)$ must be 0 .

Now, let $t_{i}=f(1,1, \ldots, 1,0,0, \ldots, 0)$ where the number of 1 s equals $i$. We proved $t_{0}=0$. If $t_{n-1}=0$, consider the bid vector where everyone bids 1 . The total VCG payment is $m$ and the total redistribution payment is $n f(1,1, \ldots, 1)=$ $n t_{n-1}=0$. This corresponds to $0 \%$ redistribution, which is contrary to our assumption that $\hat{k}>k^{*} \geq 0$. Now, consider $j=\min \left\{i \mid t_{i} \neq 0\right\}$ (which is well-defined because $t_{n-1} \neq 0$ ). If $j>m$, the property is satisfied. If $j \leq m$, consider the bid vector where $\hat{v}_{i}=1$ for $i \leq j$ and $\hat{v}_{i}=0$ for all other $i$. Under this bid vector, the first $j$ agents each get redistribution payment $t_{j-1}=0$, and the remaining $n-j$ agents each get $t_{j}$. Thus, the total redistribution payment is $(n-j) t_{j}$. Because the total VCG payment for this bid vector is 0 , we must have $(n-j) t_{j}=0$. So $t_{j}=0(j \leq$ $m<n)$. But this is contrary to the definition of $j$. Hence $f(1,1, \ldots, 1,0,0, \ldots, 0)=0$ if the number of 1 s is less than or equal to $m$.

Claim 6. $f$ satisfies the following inequalities:

$$
\begin{gathered}
\hat{k} m \leq(n-m-1) t_{m+1} \leq m \\
\hat{k} m \leq(m+i) t_{m+i-1}+(n-m-i) t_{m+i} \leq m \text { for } \\
i=2,3, \ldots, n-m-1 \\
\hat{k} m \leq n t_{n-1} \leq m
\end{gathered}
$$

Here $t_{i}$ is defined as in the proof of Claim 5. 
Proof of Claim. For $j=m+1, \ldots, n$, consider the bid vectors where $\hat{v}_{i}=1$ for $i \leq j$ and $\hat{v}_{i}=0$ for all other $i$. These bid vectors together with the non-deficit constraint and worst-case constraint produce the above set of inequalities: for example, when $j=m+1$, we consider the bid vector $\hat{v}_{i}=1$ for $i \leq m+1$ and $\hat{v}_{i}=0$ for all other $i$. The first $m+1$ agents each receive a redistribution payment of $t_{m}=0$, and all other agents each receive $t_{m+1}$. Thus, the total VCG redistribution is $(n-m-1) t_{m+1}$. The nondeficit constraint gives $(n-m-1) t_{m+1} \leq m$ (because the total VCG payment is $m$ ). The worst-case constraint gives $(n-m-1) t_{m+1} \geq \hat{k} m$. Combining these two, we get the first inequality. The other inequalities can be obtained in the same way.

We now observe that the inequalities in Claim 6 , together with $\hat{k} \geq k^{*}$, are the same as those in Claim 4 (where the $t_{i}$ are replaced by the $\hat{x}_{i}$ ). Thus, we can conclude that $\hat{k}=k^{*}$, which is contrary to our assumption $\hat{k}>k^{*}$. Hence no mechanism satisfying all the listed properties has a redistribution percentage greater than $k^{*}$ in the worst case.

So far we have only talked about the case where $n \geq m+2$. For the purpose of completeness, we provide the following claim for the $n=m+1$ case.

Claim 7. For any $m$ and $n$ with $n=m+1$, the original VCG mechanism (that is, redistributing nothing) is (uniquely) worst-case optimal among all redistribution mechanisms that are deterministic, anonymous, strategy-proof, efficient and satisfy the non-deficit constraint.

We recall that when $n=m+1$, Claim 1 tells us that the only mechanism inside the family of linear redistribution mechanisms is the original VCG mechanism, so that this mechanism is automatically worst-case optimal inside this family. However, to prove the above claim, we need to show that it is worst-case optimal among all redistribution mechanisms that have the desired properties.

Proof. Suppose a redistribution mechanism exists that satisfies all of the above properties and has a worst-case performance as good as the original VCG mechanism, that is, its worst-case redistribution percentage is greater than or equal to 0 . This implies that the total redistribution payment of this mechanism is always nonnegative.

By Lemma 2, for this mechanism, there is a function $f$ : $\mathbf{R}^{\mathbf{n}-1} \rightarrow \mathbf{R}$ so that $z_{i}=f\left(\hat{v}_{1}, \hat{v}_{2}, \ldots, \hat{v}_{i-1}, \hat{v}_{i+1}, \ldots, \hat{v}_{n}\right)$ for all $i$ and all bid vectors. We will prove that $f\left(x_{1}, x_{2}, \ldots, x_{n-1}\right)$ $=0$ for all $x_{1} \geq x_{2} \geq \ldots \geq x_{n-1} \geq 0$.

First, consider the bid vector where $\hat{v}_{i}=0$ for all $i$. Here, each agent receives a redistribution payment $f(0,0, \ldots, 0)$. The total redistribution payment is then $n f(0,0, \ldots, 0)$, which should be both greater than or equal to 0 (by the above observation) as well less than or equal to 0 (using the nondeficit criterion and the fact that the total VCG payment is $0)$. It follows that $f(0,0, \ldots, 0)=0$. Now, let us consider the bid vector where $\hat{v}_{1}=x_{1} \geq 0$ and $\hat{v}_{i}=0$ for all other $i$. For this bid vector, the agent with the highest bid receives a redistribution payment of $f(0,0, \ldots, 0)=0$, and the other $n-1$ agents each receive $f\left(x_{1}, 0, \ldots, 0\right)$. By the same reasoning as above, the total redistribution payment should be both greater than or equal to 0 and less than or equal to 0 , hence $f\left(x_{1}, 0, \ldots, 0\right)=0$ for all $x_{1} \geq 0$.

Proceeding by induction, let us assume $f\left(x_{1}, x_{2}, \ldots, x_{k}\right.$, $0, \ldots, 0)=0$ for all $x_{1} \geq x_{2} \geq \ldots \geq x_{k} \geq 0$, for some $k<n-1$. Consider the bid vector where $\hat{v}_{i}=x_{i}$ for $i \leq k+1$, and $\hat{v}_{i}=0$ for all other $i$, where the $x_{i}$ are arbitrary numbers satisfying $x_{1} \geq x_{2} \geq \ldots \geq x_{k} \geq x_{k+1} \geq 0$. For the agents with the highest $k+1$ bids, their redistribution payment is specified by $f$ acting on an input with only $k$ non-zero variables. Hence they all receive 0 by induction assumption. The other $n-k-1$ agents each receive $f\left(x_{1}, x_{2}, \ldots, x_{k}, x_{k+1}, 0, \ldots, 0\right)$. The total redistribution payment is then $(n-k-1) f\left(x_{1}, x_{2}, \ldots, x_{k}, x_{k+1}, 0, \ldots, 0\right)$, which should be both greater than or equal to 0 , and less than or equal to the total VCG payment. Now, in this bid vector, the lowest bid is 0 because $k+1<n$. But since $n=m+1$, the total VCG payment is $m \hat{v}_{n}=0$. So we have $f\left(x_{1}, x_{2}, \ldots, x_{k}, x_{k+1}, 0, \ldots, 0\right)=0$ for all $x_{1} \geq$ $x_{2} \geq \ldots \geq x_{k} \geq x_{k+1} \geq 0$. By induction, this statement holds for all $k<n-1$; when $k+1=n-1$, we have $f\left(x_{1}, x_{2}, \ldots, x_{n-2}, x_{n-1}\right)=0$ for all $x_{1} \geq x_{2} \geq \ldots \geq$ $x_{n-2} \geq x_{n-1} \geq 0$. Hence, in this mechanism, the redistribution payment is always 0 ; that is, the mechanism is just the original VCG mechanism.

Incidentally, we obtain the following corollary:

Corollary 2. No VCG redistribution mechanism satisfies all of the following: determinism, anonymity, strategyproofness, efficiency, and (strong) budget balance. This holds for any $n \geq m+1$.

Proof. For the case $n \geq m+2$ : If such a mechanism exists, its worst-case performance would be better than that of the worst-case optimal linear VCG redistribution mechanism, which by Theorem 1 obtains a redistribution percentage strictly less than 1 . But Theorem 2 shows that it is impossible to outperform this mechanism in the worst case.

For the case $n=m+1$ : If such a mechanism exists, it would perform as well as the original VCG mechanism in the worst case, which implies that it is identical to the VCG mechanism by Claim 7 . But the VCG mechanism is not (strongly) budget balanced.

\section{CONCLUSIONS}

For allocation problems with one or more items, the wellknown Vickrey-Clarke-Groves (VCG) mechanism is efficient, strategy-proof, individually rational, and does not incur a deficit. However, the VCG mechanism is not (strongly) budget balanced: generally, the agents' payments will sum to more than 0 . If there is an auctioneer who is selling the items, this may be desirable, because the surplus payment corresponds to revenue for the auctioneer. However, if the items do not have an owner and the agents are merely interested in allocating the items efficiently among themselves, any surplus payment is undesirable, because it will have to flow out of the system of agents. In 2006, Cavallo [3] proposed a mechanism that redistributes some of the VCG payment back to the agents, while maintaining efficiency, strategy-proofness, individual rationality, and the non-deficit property. In this paper, we extended this result in a restricted setting. We studied allocation settings where there are multiple indistinguishable units of a single good, and agents have unit demand. (For this specific setting, Cavallo's mechanism coincides with a mechanism proposed by Bailey in 1997 [2].) Here we proposed a family of mechanisms that redistribute some of the VCG payment 
back to the agents. All mechanisms in the family are efficient, strategy-proof, individually rational, and never incur a deficit. The family includes the Bailey-Cavallo mechanism as a special case. We then provided an optimization model for finding the optimal mechanism - that is, the mechanism that maximizes redistribution in the worst case-inside the family, and showed how to cast this model as a linear program. We gave both numerical and analytical solutions of this linear program, and the (unique) resulting mechanism shows significant improvement over the Bailey-Cavallo mechanism (in the worst case). Finally, we proved that the obtained mechanism is optimal among all anonymous deterministic mechanisms that satisfy the above properties.

One important direction for future research is to try to extend these results beyond multi-unit auctions with unit demand. However, it turns out that in sufficiently general settings, the worst-case optimal redistribution percentage is 0 . In such settings, the worst-case criterion provides no guidance in determining a good redistribution mechanism (even redistributing nothing achieves the optimal worst-case percentage), so it becomes necessary to pursue other criteria. Alternatively, one can try to identify other special settings in which positive redistribution in the worst case is possible.

Another direction for future research is to consider whether this mechanism has applications to collusion. For example, in a typical collusive scheme, there is a bidding ring consisting of a number of colluders, who submit only a single bid $[10,17]$. If this bid wins, the colluders must allocate the item amongst themselves, perhaps using payments - but of course they do not want payments to flow out of the ring.

This work is part of a growing literature on designing mechanisms that obtain good results in the worst case. Traditionally, economists have mostly focused either on designing mechanisms that always obtain certain properties (such as the VCG mechanism), or on designing mechanisms that are optimal with respect to some prior distribution over the agents' preferences (such as the Myerson auction [20] and the Maskin-Riley auction [18] for maximizing expected revenue). Some more recent papers have focused on designing mechanisms for profit maximization using worst-case competitive analysis (e.g. [9, 1, 15, 8]). There has also been growing interest in the design of online mechanisms [7] where the agents arrive over time and decisions must be taken before all the agents have arrived. Such work often also takes a worst-case competitive analysis approach [14, 13]. It does not appear that there are direct connections between our work and these other works that focus on designing mechanisms that perform well in the worst case. Nevertheless, it seems likely that future research will continue to investigate mechanism design for the worst case, and hopefully a coherent framework will emerge.

\section{REFERENCES}

[1] G. Aggarwal, A. Fiat, A. Goldberg, J. Hartline, N. Immorlica, and M. Sudan. Derandomization of auctions. STOC, 619-625, 2005.

[2] M. J. Bailey. The demand revealing process: to distribute the surplus. Public Choice, 91:107-126, 1997.

[3] R. Cavallo. Optimal decision-making with minimal waste: Strategyproof redistribution of VCG payments. AAMAS, 882-889, 2006.
[4] E. H. Clarke. Multipart pricing of public goods. Public Choice, 11:17-33, 1971.

[5] B. Faltings. A budget-balanced, incentive-compatible scheme for social choice. AMEC, 30-43, 2005.

[6] J. Feigenbaum, C. Papadimitriou, and S. Shenker. Sharing the cost of muliticast transmissions. JCSS, 63:21-41, 2001.

[7] E. Friedman and D. Parkes. Pricing WiFi at Starbucks - Issues in online mechanism design. $E C$, 240-241, 2003.

[8] A. Goldberg, J. Hartline, A. Karlin, M. Saks, and A. Wright. Competitive auctions. Games and Economic Behavior, 2006.

[9] A. Goldberg, J. Hartline, and A. Wright. Competitive auctions and digital goods. SODA, 735-744, 2001.

[10] D. A. Graham and R. C. Marshall. Collusive bidder behavior at single-object second-price and English auctions. Journal of Political Economy, 95(6):1217-1239, 1987.

[11] J. Green and J.-J. Laffont. Characterization of satisfactory mechanisms for the revelation of preferences for public goods. Econometrica, 45:427-438, 1977.

[12] T. Groves. Incentives in teams. Econometrica, 41:617-631, 1973.

[13] M. T. Hajiaghayi, R. Kleinberg, M. Mahdian, and D. C. Parkes. Online auctions with re-usable goods. EC, 165-174, 2005.

[14] M. T. Hajiaghayi, R. Kleinberg, and D. C. Parkes. Adaptive limited-supply online auctions. EC, 71-80, 2004.

[15] J. Hartline and R. McGrew. From optimal limited to unlimited supply auctions. EC, 175-182, 2005.

[16] L. Hurwicz. On the existence of allocation systems whose manipulative Nash equilibria are Pareto optimal, 1975. Presented at the 3rd World Congress of the Econometric Society.

[17] K. Leyton-Brown, Y. Shoham, and M. Tennenholtz. Bidding clubs in first-price auctions. AAAI, 373-378, 2002.

[18] E. Maskin and J. Riley. Optimal multi-unit auctions. In F. Hahn, editor, The Economics of Missing Markets, Information, and Games, chapter 14, 312-335. Clarendon Press, Oxford, 1989.

[19] H. Moulin. Efficient and strategy-proof assignment with a cheap residual claimant. Working paper, March 2007.

[20] R. Myerson. Optimal auction design. Mathematics of Operations Research, 6:58-73, 1981.

[21] R. Myerson and M. Satterthwaite. Efficient mechanisms for bilateral trading. Journal of Economic Theory, 28:265-281, 1983.

[22] D. Parkes, J. Kalagnanam, and M. Eso. Achieving budget-balance with Vickrey-based payment schemes in exchanges. IJCAI, 1161-1168, 2001.

[23] W. Vickrey. Counterspeculation, auctions, and competitive sealed tenders. Journal of Finance, 16:8-37, 1961. 